\title{
HABILIDADES SOCIALES Y ACOSO ESCOLAR: UN ESTUDIO EN CENTROS DE ENSEÑANZA SECUNDARIA DE MADRID
}

\author{
SOCIAL SKILLS AND SCHOOL BULLYNG: \\ A STUDY IN SECONDARY SCHOOLS IN MADRID
}

\author{
$M^{a}$ Luisa Dueñas Buey* y María Senra Varela** \\ Universidad Nacional de Educación a Distancia
}

\section{RESUMEN}

Este artículo es el resultado de un estudio de tipo descriptivo con una muestra de adolescentes de edades comprendidas entre los 13 y 14 años con la finalidad de analizar el fenómeno del acoso escolar, así como la incidencia de ciertas habilidades sociales relevantes en relación con el mismo. Se analiza el acoso escolar a tres niveles diferenciados: bajo, medio y alto en las 9 escalas que componen el instrumento utilizado. También se analizan:

- Los niveles de acoso escolar según el sexo.

- Las habilidades sociales en función del sexo y, finalmente,

- El acoso escolar en función de las habilidades sociales.

Palabras clave: acoso escolar, habilidades sociales, niveles de acoso escolar.

\begin{abstract}
The bullying phenomenon is a critically important subject in educational settings as well as in society. The studies conducted in this line show that people with tendency to aggressive behaviour are characterized by a distinguishable profile: instability, irritability, external attribution, high levels of anxiety, low self-esteem and tendency to depression.

This paper arises from a descriptive study with a sample of adolescents (age from 13 to 14). It is focused on the analysis of the bullying phenomenon and the impact of specific social skills on it. Nine scales of bullying are analysed: harassment, intimidation, threats, coercions, social boycott, social ex-

* Profesora del Departamento MIDE II de la Facultad de Educación de la UNED. Sus líneas de investigación se centran en el ámbito del diagnóstico y la intervención educativa con especial incidencia en el campo de la educación Especial. E-mail: mduenas@edu.uned.es

** Profesora del Departamento MIDE II de la Facultad de Educación de la UNED. Sus trabajos de investigación versan actualmente sobre poblaciones especiales. E-mail: msenra@edu.uned.es
\end{abstract}


clusion, social manipulation and aggressions. Likewise, we calculated a global index of bullying, taking three different levels into account: low, medium and high.

Social skills measured by the instrument are subsumed by six dimensions: self-expression in social interactions, defence of the own rights as a consumer, expression of annoyance or disagreement, saying "no" and interrupting social interactions, asking a favour, initiating opposite-sex peer interactions.

Furthermore, we performed analysis concerning the following aspects: Levels of bullying by sex. Social skills by sex, and, finally, bullying according to social skills.

Key words: bulling, social skills, levels of bulling.

\section{Introducción}

El fenómeno del acoso escolar, y en general de la violencia en los centros escolares, es un tema que ha ido adquiriendo una gran relevancia social en los últimos años debido, sin duda, a la mayor difusión que tienen los acontecimientos por la influencia de los medios de comunicación (prensa, radio, televisión, internet, etc.), de modo que hechos puntuales, inadmisibles para nuestra sociedad, se han convertido en temas de gran actualidad.

La agresividad más que una conducta, es una propensión, un sistema de disposiciones, de motivaciones y de conductas, del que el acoso o violencia escolar viene a ser un recurso, que cuando resulta eficaz para el control del entorno y la consecución de los objetivos, se integra como forma habitual de interacción personal. Para que este tipo de conducta llegue a instalarse como habitual se debe producir la confluencia de múltiples y variados factores de diversos tipos: biológicos, personales, familiares, sociales, cognitivos, ambientales, ...

Se puede afirmar que no todos los sujetos reaccionan del mismo modo ante situaciones difíciles, adversas y complejas. Sin embargo, se podrían destacar algunas dimensiones que se manifiestan diferenciadoras para el sujeto implicado en una dinámica de agresión y victimización. Así, se puede señalar que las personas mas proclives a la violencia suelen ser más irritables, inestables, atribuyen al exterior la responsabilidad de las actuaciones en los que se ven implicados. Estudios efectuados en este sentido indican que estas personas muestran unos índices más elevados de ansiedad y baja autoestima (Senra y Manzano, 2003). El principal moderador o inhibidor individual de dichas conductas, según Caprara (1996), sería el sentimiento de culpa, entendido como la necesidad de reparación o como miedo al castigo.

Las "formas" más habituales de agresión en estudiantes de secundaria, según Ortega (1994), son: la verbal, los insultos, los rumores y motes, seguida de la psicológica, como el aislamiento y, finalmente, la agresión física (robos, daño físico, ...).

El problema de la violencia preocupa a padres, profesores y a la sociedad en general en tanto que tienen la responsabilidad educadora de los adolescentes, pero en ocasiones se tiende a responder con una cierta impotencia y se da como "normal" aceptar que entre ellos haya una cierta agresividad.

\section{Importancia de las Habilidades Sociales en la infancia y adolescencia}

El desarrollo de las habilidades sociales está teniendo una enorme relevancia en el contexto educativo, psicológico y social. Hay constatación empírica de las sólidas relaciones 
existentes entre la competencia social en la infancia y en la adolescencia, y el posterior funcionamiento social, académico y psicológico en la vida adulta. La competencia social tiene una gran incidencia tanto en el funcionamiento presente como en el futuro desarrollo del niño; incluso, las habilidades sociales permiten que el niño vaya asimilando los roles y las normas sociales (Michelson y otros, 1987).

El comportamiento interpersonal de un niño tiene un rol muy importante en la adquisición de reforzamientos sociales y culturales. Así pues, Los individuos que manifiestan habilidades sociales adecuadas funcionan mejor en el ámbito escolar, social y emocional.

La adecuada competencia social está relacionada con logros escolares y sociales superiores, y con un ajuste personal y social en la infancia y en la adolescencia. Se considera que la conducta socialmente competente durante la infancia constituye un prerrequisito para el desarrollo y elaboración del repertorio interpersonal posterior de un individuo (Kelly, 1992).

La incompetencia se relaciona con una baja aceptación, rechazo, aislamiento social por parte de los compañeros, así como con desajustes psicológicos y problemas emocionales (Hops y Greenwood, 1988; Michelson y otros, 1987).

La familia es el contexto donde se aprenden habilidades y formas competentes de actuación a lo largo de la infancia y de la adolescencia y es el que más influencia tiene sobre las conductas posteriores del individuo. Es dentro de la familia donde los niños van aprendiendo los hábitos, los valores, las actitudes y las creencias que están en consonancia con las normas de la sociedad en la que vivimos. Las influencias familiares confluyen con las de la escuela y con las del grupo de iguales, por lo que es importante considerar el papel de los tres contextos. El marco para estudiar estas relaciones puede ser la consideración de que los diferentes mundos sociales del niño se interrelacionan entre si y la socialización, según Trianes y otros (2005), se produce en el contexto de causalidades recíprocas entre los diferentes sistemas sociales. Las investigaciones realizadas sobre los factores que tienen mayor incidencia en el desarrollo de conductas agresivas son las relativas a las prácticas de crianza infantil. Precisamente, dentro del contexto familiar se pueden ubicar tres de los más importantes factores que pueden conducir decisivamente al origen y desarrollo de conductas violentas (Olweus, 1998):

- La actitud emotiva de los padres, o de las personas encargadas del niño.

- El grado de permisividad de los padres ante la conducta agresiva del niño.

- Los métodos de afirmación de la autoridad.

Es decir, el contexto familiar tiene una trascendencia capital en lo relativo al aprendizaje de las formas de relación interpersonal.

\section{Las habilidades sociales en el contexto escolar}

La escuela es un agente de socialización de capital importancia, ya que en su entorno se desarrollan las primeras interacciones sociales fuera del ámbito familiar, y está adquiriendo cada vez una mayor responsabilidad en el desarrollo de la competencia social de sus alumnos para enfrentarse a todo un conjunto de problemas sociales y personales (Goldstein y otros, 1989) y para que consigan alcanzar una satisfactoria adaptación social en los diferentes campos en los que vayan a desenvolverse. 
La escuela como institución trasmisora de los valores dominantes en la sociedad, se ha centrado, sobre todo, en la enseñanza de habilidades académicas relacionadas con el éxito escolar (matemáticas, ciencias, idiomas, etc.), relegando su función socializadora, que debe realizar junto con la familia $y$, en general, con la sociedad.

En el contexto escolar es necesario que el alumno desarrolle unas habilidades sociales fundamentales de gran importancia tanto para su rendimiento académico como para su desarrollo social (Guralnick, 1986; Parker y Asher, 1987). Aunque existe una correlación entre las habilidades sociales requeridas para actuar de forma competente en ambos dominios, hay diferencias entre ellas, ya que están condicionadas por las tareas, contextos y situaciones sociales (Schlundt y Mc Fall, 1985), de tal forma que una actuación competente en relación con el profesor no tiene porque serlo en relación con los compañeros. En el marco escolar, la presencia de conductas violentas en las relaciones interpersonales entre escolares en los últimos años han constituido una de las principales quejas tanto de los padres como de los profesores, a la vez que se vislumbra como uno de los principales problemas y objeto de intervención, en tanto que parece ser un buen predictor de la inadaptación social en la edad adulta. Por ello, en este estudio se pretende estudiar el problema del acoso escolar, así como su relación con ciertas habilidades sociales relevantes: autoexpresión en situaciones sociales, defensa de los propios derechos, expresión de enfado o disconformidad, decir no y cortar interacciones, hacer peticiones e iniciar interacciones positivas con el sexo opuesto.

Numerosos autores evocan a los padres, centros y sociedad, la responsabilidad educadora para encauzar una adecuada socialización que permita a los alumnos convertirse en adultos socialmente competentes, sustituyendo actuaciones violentas por otros modos de expresión que impliquen una adecuada competencia prosocial (Lancelotta y Vaughn, 1989).

\section{Método}

\section{Objetivos}

Analizar el acoso escolar, así como la incidencia en el mismo de ciertas habilidades sociales relevantes.

\section{Muestra}

La muestra inicialmente recogida, muestra "aceptante" según la denominación de Fox (1981), para la realización del estudio está formada por 102 alumnos, pero de ellos hubo que desechar algunos sujetos por no haber cumplimentado la totalidad de los cuestionarios o no facilitar algún dato necesario como el sexo. Por lo tanto la muestra objeto de estudio está formada por 91 sujetos de los que 52 son varones y 39 mujeres, de edades comprendidas entre los 13 y 14 años. Los datos se recogieron directamente en dos institutos de Madrid.

\section{Diseño}

Para la finalidad del estudio y en base a los objetivos que pretendemos estudiar, nuestro diseño es de naturaleza no experimental. 
Es un estudio descriptivo ya que pretendemos describir el fenómeno objeto de estudio, explorar y analizar las relaciones que lo caracterizan y definen.

Nos planteamos un estudio transversal con la técnica de la encuesta porque es la más adecuada para los fines del estudio, es una técnica que permite cierta agilidad para recoger los datos de forma colectiva con el propósito de conocer tanto las características de la población como la relación existente entre distintas variables, siendo esto lo que realmente constituye el eje de nuestro estudio. (Martínez Arias, 1995)

\section{Variables e Instrumentos}

- Variables Independientes: Para Bisquerra (1989) son aquellas variables que no están explicadas por otras variables incluidas en el modelo, sino que su variabilidad se atribuye a causas externas al mismo, en nuestro estudio son:

- El conjunto de Habilidades Sociales que conforman la competencia social del sujeto para desenvolverse en su medio social y fundamentalmente en su medio escolar donde se desarrolla buena parte de su vida social.

- Variables Dependientes: Aquellas variables que vienen explicadas por otras variables incluidas en el modelo. Las variables que explican el comportamiento de estas variables pueden ser independientes u otras variables dependientes (Bisquerra, 1989).

- Grado de Acoso y Violencia Escolar percibida y sufrida por los alumnos participantes en el estudio.

Para la recogida de datos se han utilizado dos instrumentos ya validados y de probada fiabilidad:

- Cuestionario de Acoso y Violencia Escolar (AVE) de Iñaki Piñuel y Araceli Oñate (Piñuel y Oñate, 2006). Es un autoinforme de breve y fácil aplicación para evaluar a sujetos, entre $2^{\circ}$ de Primaria y $2^{\circ}$ de Bachillerato, en diferentes aspectos del acoso escolar. El AVE nos facilita 8 escalas indicadores del acoso y la violencia escolar, 4 factores globales de acoso y 8 escalas clínicas.

- Escala de Habilidades Sociales (EHS) de Elena Gismero Gonzalez (Gismero, 2002). Es una escala que permite evaluar el desarrollo de las habilidades sociales del sujeto.

Ambos instrumentos presentan una baremación adecuada a la edad cronológica de los sujetos de la muestra objeto de estudio.

\section{Procedimiento y técnicas de análisis de datos}

La recogida de datos se ha realizado en el aula dentro del horario escolar, con la participación voluntaria de los alumnos y la supervisión y colaboración de los profesores en el momento de la aplicación, dejando a los alumnos el tiempo necesario para cumplimentar los cuestionarios de acuerdo con las necesidades individuales.

En cuanto al análisis de los datos, en un primer momento realizamos una comparación de medias de las puntuaciones del AVE entre varones y mujeres para comprobar posibles 
diferencias en el grado de acoso escolar entre ambos sexos. Después evaluamos las habilidades sociales de los sujetos. El siguiente paso fue comparar el grado de las habilidades sociales con las medias de los factores del AVE con el fin de establecer posibles diferencias en acoso escolar en función de las habilidades sociales.

\section{Descripción de resultados}

En este apartado presentamos los resultados obtenidos con los instrumentos ya reseñados y en el posterior análisis de los mismos.

\section{a) Acoso escolar}

Hay que precisar que con el instrumento AVE se identifican a los sujetos que son víctimas de acoso. Al tratarse de un instrumento de autoinforme, son los propios sujetos los que manifiestan sentirse acosados. De los datos globales obtenidos hay un $90 \%$ que se ha sentido acosado algunas veces mientras que solo un $10 \%$ no se ha sentido nunca acosado.

La prueba discrimina varios intervalos de niveles de acoso sufrido por el sujeto, pero a efectos más prácticos los agrupamos en tres rangos: Bajo (muy bajo, bajo y casi bajo), $\boldsymbol{M e}$ dio (Medio y Casi Alto) y Alto (Alto y Muy Alto). En la siguiente tabla observamos los porcentajes por niveles en cada escala:

TABLA 1. Porcentajes en cada escala según nivel de acoso.

\begin{tabular}{|l|c|c|c|}
\hline \multicolumn{1}{|c|}{ Escalas } & Bajo & Medio & Alto \\
\hline Hostigamiento & 40.7 & 46.2 & 13.2 \\
\hline Intimidación & 83.5 & 8.8 & 7.7 \\
\hline Amenazas & 86.8 & 0.0 & 13.2 \\
\hline Coacciones & 92.3 & 0.0 & 7.7 \\
\hline Bloqueo social & 85.7 & 0.0 & 14.3 \\
\hline Exclusión social & 72.5 & 20.9 & 6.6 \\
\hline Manipulación social & 46.2 & 41.8 & 12.1 \\
\hline Agresiones & 84.6 & 7.7 & 7.7 \\
\hline Indice global de acoso & 39.6 & 48.4 & 12.1 \\
\hline
\end{tabular}

Una vez analizados los datos obtuvimos los siguientes resultados agrupados en las escalas que facilita el instrumento y discriminándolos por sexo: 
TABLA 2. Medias según sexo en las escalas de acoso.

\begin{tabular}{|l|c|c|c|}
\hline \multicolumn{1}{|c|}{ Escalas } & Varon & Mujer & $\mathbf{P}<\mathbf{0 , 0 5}$ \\
\hline Índice global de acoso & 8.69 & 7.51 & 0.482 \\
\hline Intensidad de acoso & 0.96 & 0.36 & 0.129 \\
\hline Hostigamiento & 2.73 & 2.46 & 0.646 \\
\hline Intimidación & 0.62 & 0.08 & 0.016 \\
\hline Amenazas & 0.23 & 0.20 & 0.209 \\
\hline Coacciones & 0.27 & 0.03 & 0.105 \\
\hline Bloqueo social & 0.10 & 0.31 & 0.088 \\
\hline Exclusión social & 1.08 & 1.08 & 1.000 \\
\hline Manipulación social & 1.389 & 1.15 & 0.542 \\
\hline Agresiones & 2.29 & 2.31 & 0.971 \\
\hline
\end{tabular}

Se observa que tanto en el índice global de acoso como en todas las escalas indicadores de acoso, la media de las mujeres es inferior a la de los varones, excepto en la escala de "Exclusión social" que arrojan la misma puntuación ambos sexos y la puntuación en la escala "Bloqueo social" que nos indica que las mujeres sufren más bloqueo social que los varones. Al realizar la comparación de medias de estas puntuaciones obtuvimos significación estadística en la diferencia de las medias en la escala de "Intimidación", por lo que podemos deducir que la diferencia de medias en conductas de intimidación no se debe al azar y es significativamente superior en los varones que en las mujeres, siempre referidas a la edad cronológica de nuestra muestra.

$\mathrm{Al}$ realizar el agrupamiento factorial hemos encontrado también significación estadística con un $95 \%$ de intervalo de confianza en la diferencia de medias entre ambos sexos en el factor "Intimidación".

\section{b) Habilidades Sociales}

El análisis de las puntuaciones directas en habilidades sociales se realiza de acuerdo con las seis dimensiones que recoge el instrumento. El realizar el análisis hemos obtenido los siguientes resultados: 
TABLA 3. Puntuación media en los factores de Habilidades Sociales.

\begin{tabular}{|l|c|c|c|}
\hline \multicolumn{1}{|c|}{ Dimensiones } & Varones & Mujeres & Global \\
\hline I. Autoexpresión en situaciones sociales & 24.02 & 24.92 & 24.41 \\
\hline II. Defensa de los propios derechos como consumidor & 14.40 & 14.23 & 14.33 \\
\hline III. Expresión de enfado o disconformidad & 11.08 & 11.67 & 11.33 \\
\hline IV. Decir no y cortar interacciones & 15.65 & 16.18 & 15.88 \\
\hline V. Hacer peticiones & 15.04 & 15.23 & 15.12 \\
\hline VI. Iniciar interacciones positivas con el sexo opuesto & 13.38 & 13.36 & 13.37 \\
\hline Puntuación directa global en habilidades sociales & - & - & 94.44 \\
\hline
\end{tabular}

Hay una gran similitud en las medias de ambos sexos en los distintos factores. Si acaso, se aprecia una ligera superioridad de las mujeres en los factores I y III por encima de la media global, y a su vez, los varones puntúan por encima de la media global en los factores II y VI. Al realizar la comparación de medias de ambos sexos con un intervalo de confianza del 95\% para la diferencia, no hemos encontrado significación estadística en ninguno de los seis factores.

\section{c) Habilidades Sociales y Acoso Escolar}

Analizamos el Acoso Escolar en función de las Habilidades Sociales a partir de las puntuaciones directas en cada uno de los factores de habilidades sociales y en cada una de las escalas de acoso escolar. Para el análisis agrupamos a los sujetos cuya puntuación en cada uno de los factores de Habilidades Sociales está por encima o por debajo de la media global en ese factor y procedemos a comparar las medias de ambos grupos, realizando este proceso en todas las escalas de Acoso Escolar. En la tabla 5 se refleja la significación obtenida en esta comparación de medias.

TABLA 4. Acoso escolar según las habilidades sociales.

\begin{tabular}{|l|c|c|c|c|c|c|}
\hline \multicolumn{1}{|c|}{ Escalas de Acoso } & I & II & III & IV & V & VI \\
\hline Indice global de Acoso & 0,001 & 0,007 & 0,061 & 0,020 & 0,862 & 0,038 \\
\hline Intensidad de Acoso & 0,042 & 0,011 & 0,816 & 0,042 & 0,942 & 0,299 \\
\hline Hostigamiento & 0,013 & 0,002 & 0,244 & 0,168 & 0,915 & 0,316 \\
\hline Intimidación & 0,077 & 0,112 & 0,323 & 0,021 & 0,808 & 0,232 \\
\hline Amenazas & 0,015 & 0,046 & 0,656 & 0,046 & 0,234 & 0,072 \\
\hline Coacciones & 0,054 & 0,260 & 0,236 & 0,163 & 0,847 & 0,324 \\
\hline Bloqueo social & 0,037 & 0,013 & 0,030 & 0,207 & 0,454 & 0,236 \\
\hline Exclusión social & 0,096 & 0,392 & 0,130 & 0,241 & 0,640 & 0,059 \\
\hline Manipulación social & 0,002 & 0,109 & 0,056 & 0,259 & 0,799 & 0,237 \\
\hline Agresiones & 0,005 & 0,047 & 0,159 & 0,009 & 0,509 & 0,018 \\
\hline
\end{tabular}

$\mathrm{P}<0,05$ 
- La diferencia de medias en el factor I “Autoexpresión en situaciones sociales” es significativa en las escalas de: Agresiones, Manipulación social, Bloqueo social, Amenazas y Hostigamiento, así como en Intensidad de acoso y en el Indice global de acoso.

- En el factor II "Defensa de los propios derechos como consumidor" hay significación en la diferencia de medias para las escalas de acoso de: Agresiones, Bloqueo social, Amenazas, Hostigamiento, Intensidad de acoso e Indice global de acoso.

- El factor III "Expresión de enfado o disconformidad” presenta significación en la diferencia de medias para la escala "Bloqueo social"

- En el factor IV "Decir no y cortar interacciones” hay significación en la diferencia de medias para las escalas de acoso de: Agresiones, Amenazas, Intimidación, Intensidad de acoso e Indice global de acoso.

- En el factor V "Hacer peticiones” no se encontró significación estadística en la diferencia de medias en ningún factor de acoso.

- El factor VI “Iniciar interacciones positivas con el sexo opuesto” presenta significación en la diferencia de medias para el factor Agresiones y para el Indice global de acoso.

\section{Conclusiones}

A partir del análisis de los resultados obtenidos podemos afirmar:

En primer lugar, que en el índice general de acoso el mayor porcentaje de individuos sufren acoso a un nivel medio que se caracteriza por unos componentes elevados de hostigamiento y manipulación social, y en menor porcentaje de exclusión social. Se manifiestan por el desprecio y falta de respeto por la dignidad del sujeto acosado, materializada como desprecio, burla, motes, crueldad, etc. Mediante la manipulación social el acosador distorsiona la imagen social del niño para predisponer a los demás contra él e inducirles al rechazo hacia el sujeto acosado. Estas conductas de acoso persiguen la exclusión social del sujeto, aislarlo impidiendo su participación y segregarle socialmente. En el índice global de acoso hay un $12 \%$ de niños que sufre un nivel alto de acoso, y además de los componentes anteriores hay que destacar el porcentaje de niños que sufren amenazas de otros compañeros y son objeto de manipulación social con el objeto de distorsionar su imagen y aislarlo.

En segundo lugar, aunque no hay diferencias significativas entre las medias en el índice global de acoso entre sexos, si podemos destacar la media más alta de los varones, tanto del índice global de acoso como de casi todas las escalas, destacando la escala de intimidación en la que los varones puntúan significativamente más alto que las mujeres. Las conductas de hostigamiento y coacciones también son más frecuentes en el sexo masculino, mientras que es mayor el porcentaje de mujeres que han sido víctimas de bloqueo social mediante prohibiciones de jugar o pertenecer a un grupo, de hablar o comunicarse con otros o que nadie hable o se relacione con ellas.

En tercer lugar, del análisis de los resultados obtenidos en los factores de la escala de habilidades sociales se observa que las mujeres obtienen mejor puntuación en los factores que implican expresión de sentimientos y componentes verbales, tienen más habilidad que los varones para la autoexpresión en situaciones sociales y se manejan mejor para expresar 
su enfado o disconformidad o para decir no y cortar interacciones; también son más habilidosas que los varones para hacer peticiones. Por otra parte, los varones obtienen una mayor puntuación en la habilidad de defensa de los propios derechos como consumidor y también para iniciar interacciones positivas con el sexo opuesto.

En cuarto lugar, hay que destacar la alta relación de dos factores de las Habilidades Sociales Autoexpresión en situaciones sociales" y "Defensa de los propios derechos como consumidor" con casi todos los aspectos del acoso escolar, incluido el índice global de acoso y la intensidad del mismo. Es decir, que los sujetos que habían sido víctimas de agresiones, amenazas, hostigamiento, manipulación social y bloqueo social, presentaban una capacidad más baja para expresarse de forma espontánea en distintos tipos de situaciones sociales y para la expresión de conductas asertivas en defensa de los propios derechos. Dicho de otro modo, la carencia o baja capacidad de ambos factores de las HHSS convierte a los sujetos en víctimas de agresiones, amenazas, hostigamiento, manipulación social, bloqueo social, etc.

Las mujeres tienen más capacidad de expresar disconformidad, de manifestar desacuerdo con otras personas o sentimientos negativos justificados y, a su vez, sufren en mayor medida bloqueo social con el fin de excluirlas y aislarlas impidiendo su participación y segregándolas socialmente.

Los sujetos con baja capacidad de decir no y cortar interacciones, son víctimas con mayor frecuencia e intensidad de agresiones, amenazas, intimidación, etc. Es decir que la capacidad de decir no y cortar interacciones con personas que no se desea seguir manteniendo relación, negarse a prestar o recibir algo, en general decir no a otras personas, parece ser un factor protector contra diversos aspectos de acoso escolar.

La mayor o menor capacidad para hacer peticiones a otras personas de algo que deseamos no parece tener ninguna relación significativa con el hecho de sufrir acoso escolar en cualquiera de sus aspectos.

Pero, al menos en la etapa cronológica y escolar de la muestra que estudiamos, los sujetos con baja capacidad (o falta de la misma) para iniciar interacciones con el sexo opuesto, una conversación, hablar con alguien que resulta atractivo, etc. muestran un mayor índice global de acoso y, concretamente, son con más frecuencia víctimas de agresiones.

Cabe concluir que las Habilidades Sociales actúan como factores protectores en el acoso escolar en general y en la intensidad del mismo.

\section{Referencias Bibliográficas}

Bisquerra, R. (1989). Introducción conceptual al análisis multivariable. Un enfoque informático con los paquetes SPSS-X BMPD, LISREL y SPAD. Vol. 2. Barcelona. PPU.

Caprara, G. V. (1996). "Indicadores precoces de la adaptación social”, en pp.121-145 de J. Buendía (Edt.), Psicopatología en niños y adolescentes. Desarrollos actuales. Madrid. Pirámide.

Fox, D. (1981). El proceso de investigación en educación. Pamplona. EUNSA.

Gismero González, E. (2002). Escala de habilidades Sociales. Madrid. TEA Edic.

Goldstein, A. P., Sprafkin, R. P., Gershaw, N. L. y Klein, P. (1989). Habilidades sociales y autocontrol en la adolescencia. Barcelona. Martinez Roca. 
Guralnick, M. (1986). "The peer relations of young handicapped and non handicapped children", en pp. 93-131 de P. Strain, M. Guralnick y H. Walker (Dirs.), Children's social behavior: Development assessment and modification. New York. Academic Press.

Hops, H. y Greenwood, C. R. (1988). "Social skills deficit", en J. Mash y L. G. Terdal (Eds.), Behavioral assessment of childhood disorders. New York. Guilford.

Kelly, J. A. (1992). Entrenamiento de las habilidades sociales. Bilbao. Editorial Desclée de Brouwer, $3^{\text {a }}$ edición.

Lancelotta, G. y Vaughn, S. (1989). "Relation between types of agresion and sociometric status: peer and teacher percepcions". Americam Psychological Asociation, 81 (1), pp. 86-90.

Martínez Arias, R. (1995): "El método de encuesta por muestreo: Conceptos básicos". En M. T. Anguera: Métodos de investigación en psicología. Madrid. Síntesis.

Michelson, L., Sugai, D. P., Wood, R. P. y Kazdin, A. E. (1987). Las habilidades sociales en la infancia: Evaluación y tratamiento. Barcelona. Martinez Roca.

Olweus, D. (1998). Conductas de acoso y amenazas entre escolares. Madrid. Morata.

Ortega, R. (1994). "Violencia interpersonal en los centros educativos de enseñanza secundaria. Un estudio sobre el maltrato y la intimidad entre compañeros". Revista de Educación, no 304, pp. 55-67.

Parker, J. y Asher, S. (1987). "Peer relation and later personal adjustment: Are low accepted children at risk?”. Psychological Bulletin, 102, pp. 352-390.

Piñuel, I. y Oñate, A. (2006). Acoso y Violencia Escolar. Madrid. Tea Edic.

Schlundt, D. G. y McFall, R. M. (1985). "New direction in the assessment of social competence and social skills", en pp. 22-49 de L. L'Abate y M. A. Millan (Eds.): Handbook of social skills training and research. New York. John Wiley.

Senra, M. y Manzano, N. (2003). "Inteligencia Emocional y consumo de alcohol en la adolescencia". Revista Encuentros en Psicología Social, vol. 1 (2) pp. 194-198. Actas del $8^{\circ}$ Congreso Nacional de Psicología Social. Torremolinos, Málaga, abril 2003.

Trianes, M. V., Muñoz, A. M. y Jiménez, M. (2005). Competencia social: su educación y tratamiento. Madrid. Pirámide.

Fecha de recepción: 20.07-2007

Fecha de revisión: 13-01-2009

Fecha de aceptación: 18-02-2009 